Article

\title{
Exercise-Induced Muscle Damage and Recovery in Young and Middle-Aged Males with Different Resistance Training Experience
}

\author{
John F. T. Fernandes ${ }^{1,2, *}$, Kevin L. Lamb ${ }^{2} \mathbb{D}$ and Craig Twist ${ }^{2}$ (D) \\ 1 Sport, Health and Well-being Arena, Hartpury University, Hartpury GL19 3BE, UK \\ 2 Department of Sport and Exercise Science, University of Chester, Chester CH1 4BJ, UK; \\ k.lamb@chester.ac.uk (K.L.L.); c.twist@chester.ac.uk (C.T.) \\ * Correspondence: jfmtfernandes@hotmail.co.uk
}

Received: 3 April 2019; Accepted: 23 May 2019; Published: 29 May 2019

check for updates

\begin{abstract}
This study compared the time course of recovery after a squatting exercise in trained young (YG; $n=9$; age $22.3 \pm 1.7$ years) and trained (MT; $n=9 ; 39.9 \pm 6.2$ years) and untrained (MU; $n=$ 9; age $44.4 \pm 6.3$ years) middle-aged males. Before and at 24 and $72 \mathrm{~h}$ after $10 \times 10$ squats at $60 \%$ one-repetition maximum (1RM), participants provided measurements of perceived muscle soreness (VAS), creatine kinase (CK), maximal voluntary contraction (MVC), voluntary activation (VA), and resting doublet force of the knee extensors and squatting peak power at $20 \%$ and $80 \% 1 \mathrm{RM}$. When compared to the YG males, the MT experienced likely and very likely moderate decrements in MVC, resting doublet force, and peak power at $20 \%$ and $80 \% 1 \mathrm{RM}$ accompanied by unclear differences in VAS, CK, and VA after the squatting exercise. MU males, compared to MT, experienced greater alterations in peak power at 20\% and 80\% 1RM and VAS. Alterations in CK, MVC, VA, and resting doublet force were unclear at all time-points between the middle-aged groups. Middle-aged males experienced greater symptoms of muscle damage and an impaired recovery profile than young resistance trained males. Moreover, regardless of resistance training experience, middle-aged males are subject to similar symptoms after muscle-damaging lower-body exercise.
\end{abstract}

Keywords: squatting; ageing; muscle damage

\section{Introduction}

The number of middle-aged (i.e., 30 to 59 years old) people in the U.K. is increasing [1]. Alongside this is a growing number of middle-aged athletes, many of whom want to maintain or improve their athletic performances despite the natural age-related declines [2]. Specifically, these impairments are because of losses in muscle mass [3] and strength and power [3,4], of which, the lower-body undergoes the greatest losses [3-5]. Importantly, resistance training can provide a potent method of ameliorating these age-associated losses in muscle mass, strength, and power [6].

When used acutely, resistance exercise can cause exercise-induced muscle damage (EIMD; [6]), for which the mechanisms have been discussed extensively before (see [7]). EIMD symptoms include increases in muscle soreness, intramuscular enzymes in the blood serum, and plasma, and, of most importance to the athlete, an impaired muscle function [8]. Importantly, changes in muscle function provide the best indication of EIMD [7,8]. Although highly individualised [9], these symptoms typically peak between 24 and $48 \mathrm{~h}$ after the initial bout and are recovered by seven days [7]. A muscle's susceptibility to damage might also be affected (reduced) in subsequent bouts where prior eccentric exercise has occurred $[10,11]$. Two studies have noted that this protection from eccentric exercise is less pronounced ( 29\% in MVC) in untrained older, compared to younger, men [12,13], which suggests 
that older resistance-trained men might exhibit symptoms of EIMD that are not dissimilar to their untrained counterparts.

Studies examining the recovery of older and younger untrained adults after muscle-damaging exercise are equivocal. Some studies have reported greater symptoms of EIMD in younger, compared to older, males [14,15], while others have observed greater EIMD in older ( $\sim 99$ to 66 years), compared to younger, males ( 23 years) (17). Moreover, a number of studies have reported no difference in symptoms of EIMD after exercise for young populations ( 19 years), compared to older populations ( $\sim 48$ to 76 years) $[6,16-19]$. One confounding factor in the current literature might be the physical activity and resistance training status of the participants. For example, when controlling for physical activity, Buford et al. [18] noted that recovery from muscle-damaging unilateral plantar flexion was similar among young ( $\sim 23$ years) and older ( $\sim 6$ years) adults. Despite the effectiveness of resistance training in combating the age-associated losses, only one study has investigated the EIMD response in older resistance trained males. Like Buford et al. [18], Gordon and colleagues [16] observed no differences in indirect markers of EIMD between recreationally trained young ( 22 years) and middle-aged ( $\sim 7$ years) males after damaging knee extensor exercise. Despite these novel findings, no study has yet reported on the recovery characteristics from multi-jointed lower-body exercise in middle-aged (35 to 55 years), resistance trained males. Indeed, Gordon et al. [16] advised that future studies might adopt a more ecologically valid exercise protocol. Data from such a study would be highly applicable to those athletes seeking to prolong their athletic careers. Consequently, the primary aim of the study was to determine the time course to recovery from EIMD in young and middle-aged resistance trained males. A secondary purpose was to determine if the recovery profile of middle-aged males is altered by resistance training experience. Given the variability in the current data regarding EIMD and ageing and a lack of studies in trained populations, we propose the null hypothesis, i.e., that the EIMD response would not be different between groups.

\section{Materials and Methods}

\subsection{Design}

The study used a two-way repeated measures design (age group $x$ time), whereby participants attended the laboratory on four separate occasions, the initial visit for estimations of body composition and the back squat 1RM (Figure 1). On the same visit they were habituated with the measurements of squatting peak power and MVC, VA, and resting doublet force during isometric knee extension. Participants were considered 'habituated' when they could complete three consecutive repetitions that produced power or force values each within $10 \%$ [20]. Participants returned to the laboratory 2-4 days later for measurements comprising squats at $20 \%$ and $80 \% 1 \mathrm{RM}, \mathrm{MVC}$, VA, resting doublet force, muscle soreness, and creatine kinase $(\mathrm{CK})$ activity, and an exercise bout comprising $10 \times 10$ squats at $60 \%$ 1RM [21]. Repeated measures were then conducted 24 and $72 \mathrm{~h}$ after the initial exercise bout.

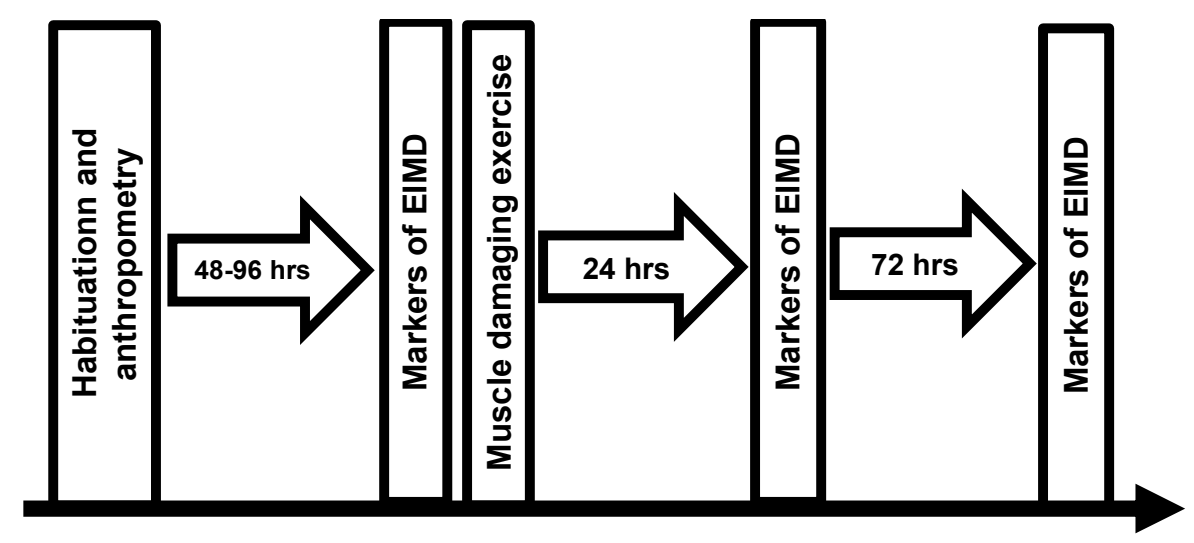

Figure 1. Schematic of study design. 


\subsection{Participants}

Nine young resistance trained (YG; range: 21 to 25 years), nine middle-aged (MT; range: 35 to 54 years) resistance trained, and nine untrained middle-age males (MU; range: 35 to 53 years) were recruited for this study using convenience sampling. Thirty-five years was selected as the lower boundary for the middle-aged group because it is the entry age for 'Masters' athletes (see British Masters Athletic Federation and World Masters Athletics). As age-related studies typically use older groups (60 years and over), 55 was selected as the upper-limit for the middle-aged group. An overall sample size of approximately 27 (nine per group) was estimated using Batterham and Atkinson's [22] nomogram. This was calculated using a coefficient of variation and typical change of $6.1 \%$ [23] and 5\%, respectively. The YG and MT had a minimum of two years' resistance training experience and regularly used squats as part of their resistance training programmes. The MU group had no resistance training experience, but was screened by the lead researcher to ensure they could perform the correct squat technique. All participants had been active in sport for a minimum of two years and were competitive. Participants completed a pre-test health questionnaire and provided written consent for the study, which was approved by the Ethics Committee of the Faculty of Life Sciences at the host institution. Participants were instructed not to consume any ergogenic supplements (for example, caffeine) on the day of testing and to refrain from exercise, other than that performed as part of the study, throughout their involvement.

\subsection{Procedures}

\subsubsection{Anthropometric Measurements}

Body density was estimated via skinfold thickness measurements (Harpenden, British Indicators, Burgess Hill, UK) taken at the triceps, axilla, abdominal, suprailiac, chest, subscapular, and mid-thigh [24]. Body fat percentage (\%BF) was estimated [25] from which quantities (kg) of fat-mass (FM) and fat-free mass (FFM) were derived.

\subsubsection{Resistance Training History and Sports Participation}

The YG and MT participants completed a questionnaire to record how many years they had participated in regular resistance training, their weekly training frequency and session duration, and the main reason for their training. A second questionnaire detailed how many years they had participated in organised sport, their weekly frequency and session duration, and the type of sport they in which participated (i.e., team, endurance, racket, or other).

\subsubsection{Maximal Strength Testing}

The 1RM for squat exercise was predicted using a three-repetition maximum (3RM) protocol. Participants performed 8-10 repetitions with $50 \%$ of their estimated $1 \mathrm{RM}$, followed by $3-5$ repetitions with $85 \%$ of their estimated 1RM. The load was then set at the approximate 3RM and the participants performed three repetitions. The load was progressively increased until the participant could no longer perform a complete repetition. The final load lifted was then used with the following equation [26] to estimate the 1RM squat load:

$$
1 \mathrm{RM}=(100 \times 3 \mathrm{RM} \text { load lifted }) /\left[48.8+\left(53.8 \times 2.71828^{-0.075} \times \text { repetitions }\right) .\right.
$$

The above equation has been reported to yield accurate 1RM predictions $(r=0.969,0.02 \%$ different from direct 1RM) [27].

\subsubsection{Indirect Markers of Muscle Damage}

Perceived muscle soreness of the knee extensors was measured using a 0-10 visual analogue scale (VAS). Plasma CK activity was also determined from a capillary blood sample. A $30 \mu \mathrm{L}$ sample of 
whole blood was collected into a heparinised capillary tube and pipetted onto a test strip for analysis (Reflotron, Type 4, Boehringer Mannheim, Mannheim, Germany).

\subsubsection{Assessment of Maximal Voluntary Contraction and Voluntary Activation}

Before undertaking the MVC and VA assessments, participants performed a warm-up comprising five minutes of cycling at $100 \mathrm{~W}$ (Lode, Corival, Groningen, Netherlands). An isometric dynamometer (Biodex, Multi-joint system 3, Biodex Medical, New York, NY, USA) was employed to measure the force of the participant's dominant knee extensor at $80^{\circ}$ knee flexion. To prevent extraneous body movements, Velcro straps were applied tightly across the chest and thigh. Participants were provided with strong verbal encouragement and real-time feedback via the PC monitor.

The knee extensors were electrically stimulated ( $5 \mathrm{~s}$ with two $100 \mathrm{~Hz}$ single square impulses (doublet); Digitimer, D57, Hertfordshire, UK) using two $5 \times 13 \mathrm{~cm}$ moistened surface electrodes (Axelgaard Manufacturing Co., Ltd., Fallbrook, CA, USA); one placed distally over the quadriceps and the other proximally over the upper quadricep. During optimisation, the amplitude of a doublet was progressively increased, starting at $50 \mathrm{amps}$, until a point where no further increases in intensity resulted in an increase in resting doublet force. Initially, a 230 volt electrically evoked doublet (set $20 \%$ above the value required to evoke a resting muscle doublet of maximum amplitude) was applied to the resting muscle (resting doublet) at $1 \mathrm{~s}$. The resting doublet was used to elucidate any peripheral alterations that might have occurred as a result of the squatting protocol [21]. Participants then performed a $4 \mathrm{~s} \mathrm{MVC} \mathrm{before} \mathrm{a} \mathrm{doublet,} \mathrm{which} \mathrm{was} \mathrm{applied} \mathrm{at} \mathrm{the} \mathrm{isometric} \mathrm{plateau} \mathrm{(superimposed}$ doublet). The MVC was taken as the average force over $50 \mathrm{~ms}$ (AcqKnowledge 3 software, Biopac Systems, Massachusetts, MA, USA) before the superimposed doublet was applied. VA was calculated according to the interpolated doublet ratio using the equation:

$$
\text { VA }(\%)=[1-(\text { size of superimposed doublet } / \text { size of resting doublet })] \times 100 \text {. }
$$

A similar procedure has been deemed a reliable method $(\mathrm{CV}=3.38 \%)$ for assessing VA [28].

\subsubsection{Assessment of Peak Power During Squat}

Peak power was assessed at loads corresponding to $20 \%$ and $80 \% 1 \mathrm{RM}$ during the back squat exercise using a rotary encoder (FitroDyne, Fitronic, Bratislava, Slovakia), the procedures for which have been described elsewhere $[5,23]$. The FitroDyne has been shown to produce reliable intra- and inter-day measures of peak power (coefficient of variation $=3.9-6.1 \%$ ) at the selected loads [23].

\subsubsection{Muscle-Damaging Exercise Protocol}

This consisted of $10 \times 10$ repetitions of squat exercise at a load corresponding to $60 \% 1 \mathrm{RM}$ with $120 \mathrm{~s}$ rest between sets [21]. Each repetition was performed in the manner outlined above. A similar protocol has successfully induced symptoms of muscle damage in previous research [21,29]. The FitroDyne was used to calculate the power for each repetition in the manner outlined above. The average peak power per repetition was used to elucidate the influence of exercise intensity on recovery profiles between groups. One participant from the MU group was unable to complete sets 8,9 , and 10 at $60 \% 1 \mathrm{RM}$, thus the load was reduced by $5 \mathrm{~kg}(50.1 \% 1 \mathrm{RM})$ and power values were calculated accordingly.

\subsection{Statistical Analyses}

Comparisons of categorical training history and sport participation variables by group were made using a chi-squared $\left(\chi^{2}\right)$ test of association. All other data were analysed using the effect size (ES) with $90 \%$ confidence intervals (CI) [30]. Magnitude-based inference statistics were used to provide information on the size of the differences, allowing for a more practical and meaningful explanation of the data [31]. Thresholds for the magnitude of the observed change for each variable were determined as the within-participant standard deviation in that variable $\times 0.2,0.6,1.2$, and 2 for a small, moderate, 
large, and very large effect [32]. Threshold probabilities for a meaningful effect, based on the $90 \%$ confidence limits (CL) were as follows: Less than $0.5 \%$ most unlikely, $0.5-5 \%$ very unlikely, $5-25 \%$ unlikely, $25-75 \%$ possibly, $75-95 \%$ likely, $95-99.5 \%$ very likely, and $>99.5 \%$ most likely. Effects with confidence limits across a likely small positive or negative change were classified as unclear [30]. All calculations were completed using predesigned spreadsheets (www.sportsci.org). Data are presented as ES, lower $\mathrm{CI}$, and upper CI.

\section{Results}

\subsection{Biometric Measures and Training History}

Age and sum of skinfolds were most likely and likely higher, respectively, in the MT groups compared to the YG group (Table 1). Differences in FM and body fat percentage between the YG and MT groups were very likely, while mass and squat 1RM were unclear. Age and FFM differences between the MT and MU groups were likely moderate, whilst all other biometric characteristics demonstrated unclear differences.

The MT group had most likely regularly resistance trained for longer than the YG (ES 2.29, CI 1.46, 3.13; Table 2), though their training was associated with a lower weekly frequency $\left(\chi^{2}=32.5, p<0.05\right)$ and shorter session duration $\left(\chi^{2}=36.4, p<0.05\right)$. Moreover, the MT group typically chose resistance training for strength and fat loss, whereas the YG trained for strength $\left(\chi^{2}=31.8, p<0.05\right)$.

Table 1. Biometric characteristics (mean $\pm \mathrm{SD}$ ) and comparisons of young (YG) and middle-aged trained (MT) and untrained (MU) groups.

\begin{tabular}{|c|c|c|c|c|c|}
\hline \multirow{2}{*}{ Measure } & \multicolumn{3}{|c|}{ Group } & \multicolumn{2}{|c|}{ Comparison } \\
\hline & YG $(n=9)$ & MT $(n=9)$ & MU $(n=9)$ & YG v MT & MT v MU \\
\hline Age (years) & $22.3 \pm 1.7$ & $39.9 \pm 6.2$ & $44.4 \pm 6.3$ & $\begin{array}{c}\text { Most likely } \uparrow \\
3.70(2.87,4.53)\end{array}$ & $\begin{array}{c}\text { Likely } \uparrow \\
0.71(-0.10,1.52)\end{array}$ \\
\hline Mass (kg) & $82.0 \pm 9.0$ & $79.1 \pm 10.3$ & $83.4 \pm 9.56$ & $\begin{array}{c}\text { Unclear } \\
0.29(-1.10,0.52)\end{array}$ & $\begin{array}{c}\text { Unclear } \\
0.42(-0.39,1.23)\end{array}$ \\
\hline $\begin{array}{l}\text { Fat-free mass } \\
\qquad(\mathrm{kg})\end{array}$ & $71.4 \pm 7.9$ & $63.9 \pm 6.5$ & $68.6 \pm 7.1$ & $\begin{array}{c}\text { Very likely } \downarrow \\
-1.02(-1.83,-0.22)\end{array}$ & $\begin{array}{c}\text { Likely } \uparrow \\
0.68(-0.13,1.49)\end{array}$ \\
\hline Fat-mass (kg) & $10.5 \pm 4.5$ & $15.2 \pm 5.7$ & $14.8 \pm 7.0$ & $\begin{array}{c}\text { Likely } \uparrow \\
0.89(0.09,1.70)\end{array}$ & $\begin{array}{c}\text { Unclear } \\
-0.07(-0.88,0.74)\end{array}$ \\
\hline Body fat (\%) & $12.8 \pm 4.7$ & $18.8 \pm 5.8$ & $17.4 \pm 6.7$ & $\begin{array}{c}\text { Very likely } \uparrow \\
1.13(0.32,1.94)\end{array}$ & $\begin{array}{c}\text { Unclear } \\
-0.23(-1.04,0.58)\end{array}$ \\
\hline $\begin{array}{c}\text { Sum of } \\
\text { skinfolds (mm) }\end{array}$ & $82.3 \pm 24.6$ & $102.4 \pm 31.9$ & $91.7 \pm 32.7$ & $\begin{array}{c}\text { Likely } \uparrow \\
0.69(-0.12,1.50)\end{array}$ & $\begin{array}{c}\text { Unclear } \\
-0.32(-1.13,0.48)\end{array}$ \\
\hline Squat 1RM (kg) & $130.8 \pm 26.8$ & $109.3 \pm 22.5$ & $98.4 \pm 14.25$ & $\begin{array}{c}\text { Unclear } \\
-0.85(-1.65,-0.04)\end{array}$ & $\begin{array}{c}\text { Unclear } \\
-0.56(-1.37,0.25)\end{array}$ \\
\hline
\end{tabular}

The comparison panel details the qualitative descriptor, effect size, and upper and lower confidence limits.

Table 2. Resistance training characteristics of the young (YG) and middle-aged trained groups (MT).

\begin{tabular}{|c|c|c|c|}
\hline \multicolumn{2}{|c|}{ Resistance Training Characteristics } & YG $(n=9)$ & MT $(n=9)$ \\
\hline \multicolumn{2}{|c|}{ Years of resistance training (mean $\pm \mathrm{SD}$ ) } & $4.6 \pm 1.3$ & $18.0 \pm 5.6$ \\
\hline \multirow{3}{*}{ Weekly frequency * } & 1 to 2 & $2(22.2)$ & $6(66.7)$ \\
\hline & 3 to 4 & $4(44.4)$ & $2(22.2)$ \\
\hline & $5+$ & $3(33.3)$ & $1(11.1)$ \\
\hline \multirow{4}{*}{ Session duration * } & 0 to $30 \mathrm{~min}$ & $0(0.0)$ & $1(11.1)$ \\
\hline & 31 to $60 \mathrm{~min}$ & $3(33.3)$ & $7(77.8)$ \\
\hline & 61 to $90 \mathrm{~min}$ & $5(55.6)$ & $1(11.1)$ \\
\hline & $90+\min$ & $1(11.1)$ & $0(0.0)$ \\
\hline \multirow{4}{*}{ Reason for resistance training * } & Strength & $6(66.7)$ & $4(44.4)$ \\
\hline & Hypertrophy & $1(11.1)$ & $0(0.0)$ \\
\hline & Fat loss & $1(11.1)$ & $4(44.4)$ \\
\hline & Health & $1(11.1)$ & $1(11.1)$ \\
\hline
\end{tabular}

* Categorical variables are significantly associated $(p<0.05)$. Brackets denote percentage of responses in each category. 
There were very likely large and moderate differences in sports participation for the MT compared to the YG and MU, respectively, with MT having more years compared to the YG (ES 1.47, CI 0.66, 2.28) and less than the MU group (ES 1.17, CI 0.36, 1.98; Table 3). No relationship ( $p>0.05$ ) was observed between groups for weekly frequency, session duration, or type of sport played.

Table 3. Sports participation characteristics of the young and middle-aged trained groups.

\begin{tabular}{llccc}
\hline \multicolumn{1}{c}{ Sports Participation Characteristics } & YG $(\boldsymbol{n}=\mathbf{9 )}$ & MT $(\boldsymbol{n}=\mathbf{9 )}$ & MU $(\boldsymbol{n}=\mathbf{9})$ \\
\hline Years of sports participation (mean \pm SD) & $11.2 \pm 4.8$ & $22.0 \pm 7.8$ & $30.3 \pm 7.8$ \\
\hline \multirow{3}{*}{ Weekly frequency } & 1 to 2 & $4(44.4)$ & $2(22.2)$ & $0(0.0)$ \\
& 3 to 4 & $4(44.4)$ & $4(44.4)$ & $6(66.7)$ \\
& $5+$ & $1(11.1)$ & $3(33.3)$ & $3(33.3)$ \\
\hline \multirow{5}{*}{ Session duration } & 0 to $30 \mathrm{~min}$ & $0(0.0)$ & $0(0.0)$ & $0(0.0)$ \\
& 31 to $60 \mathrm{~min}$ & $3(33.3)$ & $4(44.4)$ & $7(77.8)$ \\
& 61 to $90 \mathrm{~min}$ & $3(33.3)$ & $3(33.3)$ & $1(11.1)$ \\
& $90+$ min & $3(33.3)$ & $2(22.2)$ & $1(11.1)$ \\
\multirow{5}{*}{ Type of sport } & Team & $5(55.6)$ & $3(33.3)$ & $3(33.3)$ \\
& Endurance & $3(33.3)$ & $5(55.6)$ & $4(44.4)$ \\
& Racket & $0(0.0)$ & $1(11.1)$ & $2(22.2)$ \\
& Other & $1(11.1)$ & $0(0.0)$ & $0(0.0)$ \\
\hline
\end{tabular}

\subsection{External Load Response during the Muscle-Damaging Protocol}

There was a likely moderate lower average peak power (ES -0.71 CI -1.53, 0.10) in the MT $(603.2 \pm$ $162.6 \mathrm{~W})$ compared to the YG $(770.4 \pm 278.0 \mathrm{~W})$. Differences between the MT and MU $(547.0 \pm 75.0 \mathrm{~W})$ groups were unclear (ES $-0.43, \mathrm{CI}-1.25,0.39$ ).

\subsection{Indirect Markers of Muscle Damage}

At Pre, differences in muscle soreness between the YG and MT and MT and MU were unclear (ES 0.00, CI -0.81, 0.81 and ES 0.42, CI -0.39, 1.22, respectively; Figure 2). When the three groups were combined, perceived muscle soreness demonstrated most likely very large (ES 4.20, CI 3.74, 4.65) increases at $24 \mathrm{~h}$ and, likewise (ES 1.82, CI 1.36, 2.27), at $72 \mathrm{~h}$ after muscle-damaging exercise. Between-group differences for the YG and MT comparison were unclear at 24 and $72 \mathrm{~h}$ after muscle-damaging exercise. Increases in muscle soreness were likely moderately higher in the MU group compared to the MT group at 24 and $72 \mathrm{~h}$.

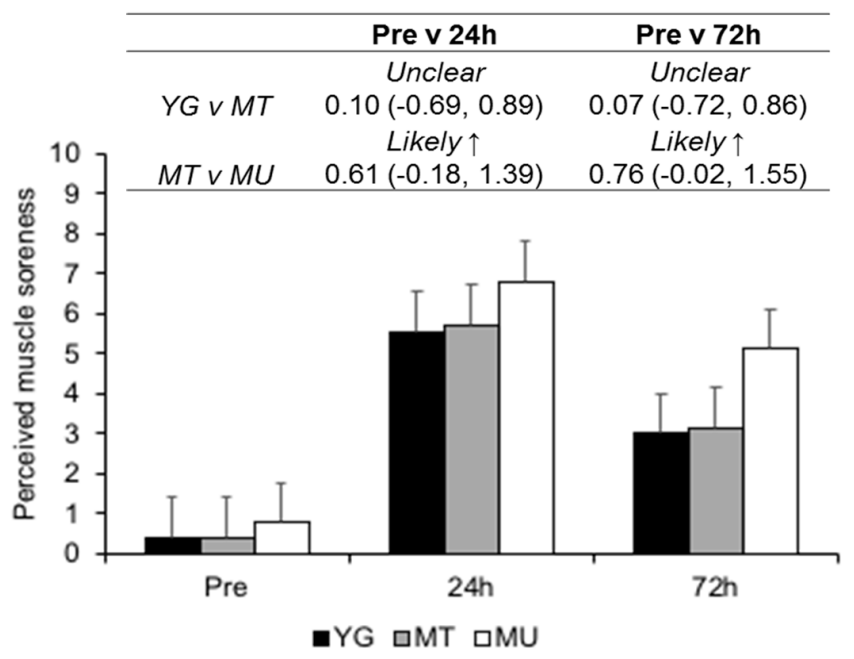

Figure 2. Changes in perceived muscle soreness between YG, MT, and MU at pre, 24, and $72 \mathrm{~h}$ after resistance exercise. The panel above details the qualitative descriptor, effect size, and upper and lower confidence limits. 
Differences in CK activity at Pre for YG and MT and MT and MU comparisons were unclear (ES -0.41, CI -1.21, 0.40 and ES -0.44, CI -1.25, 0.38, respectively; Figure 3). The increase in plasma CK activity for the three groups combined was very likely moderate (ES 1.19, CI 0.73, 1.64) and likely small (ES 0.59, CI 0.13, 1.05) at 24 and $72 \mathrm{~h}$, respectively, compared to Pre. Differences in plasma CK activity over time were unclear between the YG and MT groups. Plasma CK activity was likely moderately higher in the MU group compared to the MT group at $24 \mathrm{~h}$, though differences between the groups were unclear at $72 \mathrm{~h}$.

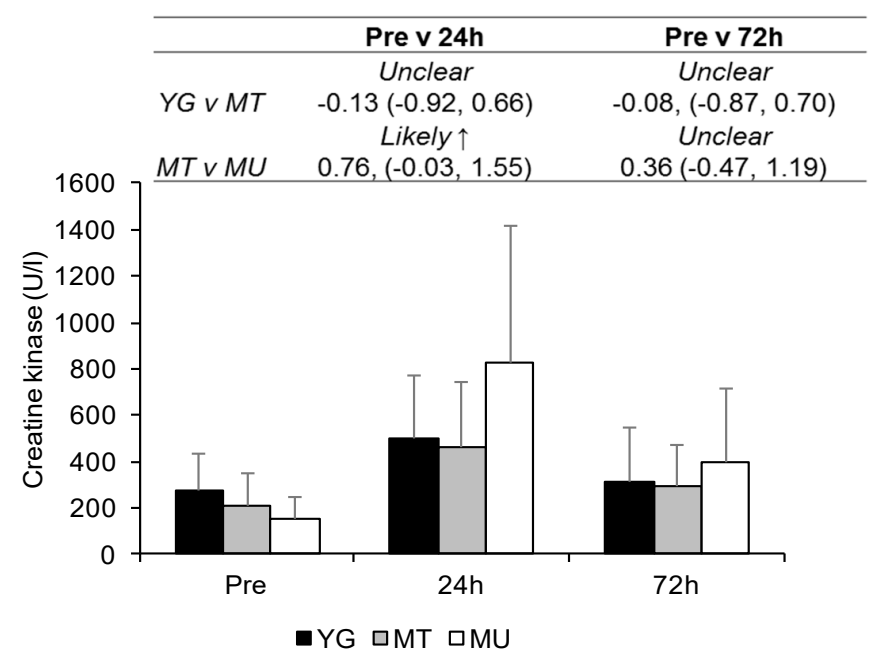

Figure 3. Changes in plasma creatine kinase activity between YG, MT, and MU at Pre, 24, and $72 \mathrm{~h}$ after resistance exercise. The panel above details the qualitative descriptor, effect size, and upper and lower confidence limits.

At Pre, differences in MVC force were likely moderate and unclear for the YG compared to MT (ES -0.80, CI -1.61, 0.01) and MT compared to MU (ES 0.27, CI -0.56, 1.10), respectively (Figure 4). MVC force had very likely moderate (ES -0.71, CI -1.16, -0.26) and likely small (ES -0.39, CI -0.84, 0.06) decreases at 24 and $72 \mathrm{~h}$ after muscle-damaging exercise. Likely and very likely moderate reductions in MVC force were observed in the MT group compared to the YG groups at 24 and $72 \mathrm{~h}$, respectively. At 24 and $72 \mathrm{~h}$, differences between the MT and MU groups were unclear.

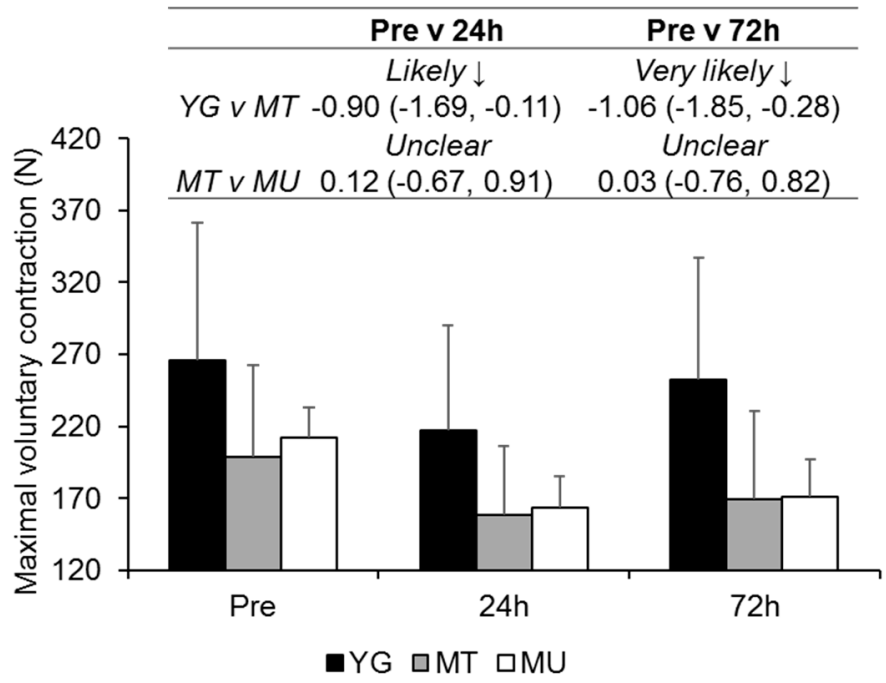

Figure 4. Changes in maximal voluntary contraction force between YG, MT, and MU at Pre, 0, 24, and $72 \mathrm{~h}$ after resistance exercise. The panel above details the qualitative descriptor, effect size, and upper and lower confidence limits. 
Differences in VA at Pre were unclear for YG compared to MT (ES 0.03, CI -0.77, 0.84) and MT compared to MU (ES 0.07, CI -0.76, 0.90; Figure 5). When all groups were combined VA decreased over time, with values at 24 and $72 \mathrm{~h}$ demonstrating very likely moderate decreases (ES -0.87, CI -1.33, -0.41 and ES $-0.88, \mathrm{CI}-1.34,-0.41$, respectively). Differences between groups were unclear at all time-points.

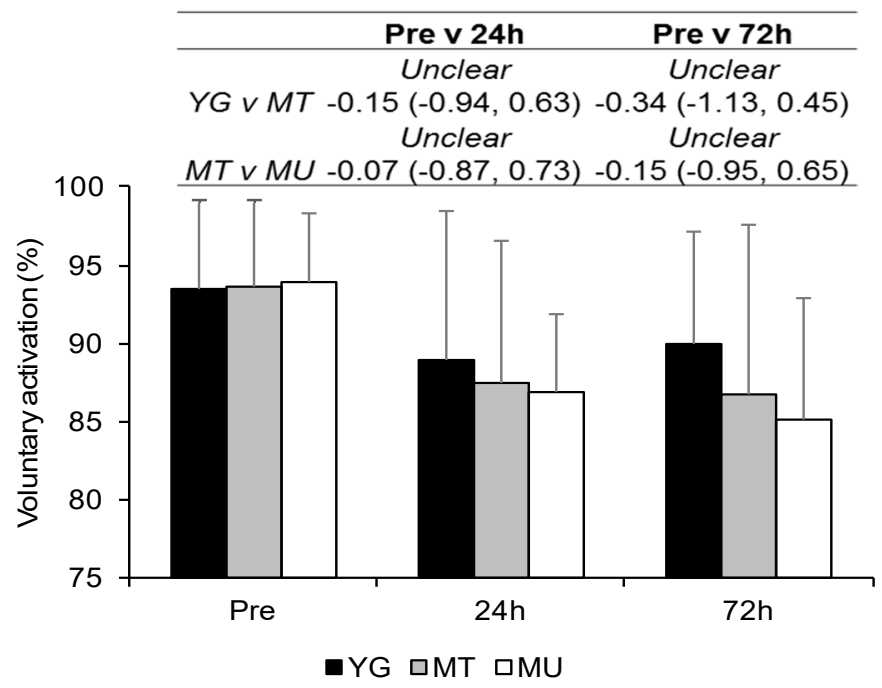

Figure 5. Changes in voluntary activation between YG, MT and MU at Pre, 24, and $72 \mathrm{~h}$ after resistance exercise. The panel above details the qualitative descriptor, effect size, and upper and lower confidence limits.

Higher mean resting doublet values for the YG were likely moderate compared to the MT (ES -0.96 CI -1.77, 0.14; Figure 6). Similarly, higher values for MU (ES 0.95, CI 0.12, 1.78) were likely moderate compared to the MT group. Mean doublet values were likely small and unclear at 24 and $72 \mathrm{~h}$, respectively, (ES $-0.52, \mathrm{CI}-0.98,-0.06$ and ES $-0.04, \mathrm{CI}-0.50,0.42$, respectively) after squatting exercise. Differences in resting doublet were very likely moderate and likely moderate between YG and MT groups at 24 and $72 \mathrm{~h}$, respectively. MT and MU comparisons were unclear at 24 and $72 \mathrm{~h}$.

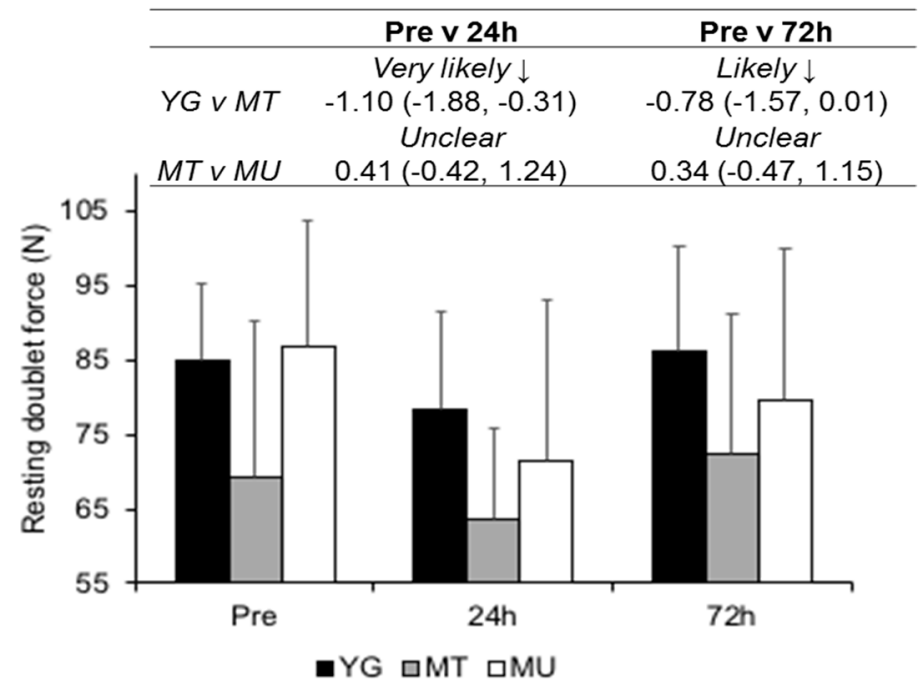

Figure 6. Changes in resting doublet force between YG, MT and MU at Pre, 24, and $72 \mathrm{~h}$ after resistance exercise. The panel above details the qualitative descriptor, effect size, and upper and lower confidence limits. 


\subsection{Peak Power during Squat Exercise}

At Pre, a very likely moderate lower peak power was at 20\% and 80\% 1RM (ES -1.03, CI -1.84, -0.22 and ES $-1.03, \mathrm{CI}-1.84,-0.21$, respectively) was observed in the MT compared to YG (Table 4). Differences at Pre for MT and MU were most likely very large and unclear for $20 \%$ and $80 \% 1 \mathrm{RM}$ (ES -3.34, $\mathrm{CI}-4.18,-2.50$ and ES $-0.47, \mathrm{CI}-1.28,0.33$, respectively). When all groups were combined, peak power for $20 \%$ and $80 \% 1 \mathrm{RM}$ demonstrated possibly small (ES $-0.25, \mathrm{CI}-0.71,0.20$ and ES $-0.36, \mathrm{CI}-0.81,0.09$, respectively) and unclear (ES $-0.23, \mathrm{CI}-0.69,0.22$ and ES $-0.19, \mathrm{CI}-0.64,0.26$, respectively) decrements at 24 and $72 \mathrm{~h}$, respectively. For $20 \%$ and $80 \% 1 \mathrm{RM}$, between group differences at 24 and $72 \mathrm{~h}$ were very likely moderate between the YG and MT groups. Similarly, reductions in 20\% 1RM peak power at 24 and $72 \mathrm{~h}$ for the MT vs. MU comparison were very likely moderate. Peak power at $80 \% 1 \mathrm{RM}$ illustrated likely moderate and very likely large differences at 24 and $72 \mathrm{~h}$, respectively.

Table 4. Peak power at Pre, 24 and $72 \mathrm{~h}$.

\begin{tabular}{|c|c|c|c|c|c|c|}
\hline \multirow{2}{*}{ Intensity } & \multirow{2}{*}{ Group } & \multirow{2}{*}{ Pre } & \multirow{2}{*}{$24 \mathrm{~h}$} & \multirow{2}{*}{$72 \mathrm{~h}$} & \multicolumn{2}{|c|}{ Comparison $(90 \% \mathrm{CI})$} \\
\hline & & & & & Pre v 24 h & Pre v $72 \mathrm{~h}$ \\
\hline \multirow{6}{*}{$\begin{array}{c}20 \% 1 \mathrm{RM} \\
(\mathrm{W})\end{array}$} & \multirow{2}{*}{ YG } & \multirow{2}{*}{$507.9 \pm 134.6$} & \multirow{2}{*}{$473.8 \pm 119.9$} & \multirow{2}{*}{$476.6 \pm 119.7$} & \multicolumn{2}{|c|}{ YG v MT } \\
\hline & & & & & Very likely $\downarrow$ & Very likely $\downarrow$ \\
\hline & \multirow{2}{*}{ MT } & \multirow{2}{*}{$387.4 \pm 87.9$} & \multirow{2}{*}{$360.3 \pm 76.1$} & \multirow{2}{*}{$366.3 \pm 76.4$} & $-1.07(-1.85,-0.28)$ & $-1.04(-1.82,-0.25)$ \\
\hline & & & & & \multicolumn{2}{|c|}{ MT v MU } \\
\hline & \multirow{2}{*}{$\mathrm{MU}$} & \multirow{2}{*}{$320.7 \pm 47.9$} & \multirow{2}{*}{$291.7 \pm 40.1$} & \multirow{2}{*}{$289.7 \pm 40.2$} & Very likely $\downarrow$ & Very likely $\downarrow$ \\
\hline & & & & & $-1.06(-1.84,-0.27)$ & $-1.17(-1.96,-0.39)$ \\
\hline \multirow{6}{*}{$\begin{array}{c}80 \% 1 \mathrm{RM} \\
(\mathrm{W})\end{array}$} & \multirow{2}{*}{ YG } & \multirow{2}{*}{$1295.3 \pm 369.1$} & \multirow{2}{*}{$1207.5 \pm 328.2$} & \multirow{2}{*}{$1275.9 \pm 338.3$} & \multicolumn{2}{|c|}{ YG v MT } \\
\hline & & & & & Very likely $\downarrow$ & Very likely $\downarrow$ \\
\hline & \multirow{2}{*}{ MT } & \multirow{2}{*}{$977.1 \pm 211.1$} & \multirow{2}{*}{$869.8 \pm 195.0$} & \multirow{2}{*}{$964.9 \pm 212.1$} & $-1.07(-1.96,-0.39)$ & $-1.04(-1.83,-0.25)$ \\
\hline & & & & & \multicolumn{2}{|c|}{ MT v MU } \\
\hline & \multirow{2}{*}{ MU } & \multirow{2}{*}{$886.0 \pm 163.2$} & \multirow{2}{*}{$746.7 \pm 153.3$} & \multirow{2}{*}{$735.1 \pm 134.8$} & Likely $\downarrow$ & Very likely $\downarrow$ \\
\hline & & & & & $-0.67(-1.45,0.12)$ & $-1.22(-2.01,-0.43)$ \\
\hline
\end{tabular}

The comparison panel details the qualitative descriptor, effect size, and upper and lower confidence limits.

\section{Discussion}

Contrary to our hypothesis, the current findings highlight the magnitude of exercise-induced muscle damage and time-course of recovery after lower body resistance exercise is greater in trained middle-aged males than their young counterparts. Moreover, regardless of resistance training experience, middle-aged males experienced like symptoms of muscle damage and a similar recovery profile in the days after.

\subsection{Confirmation of EIMD}

The small to moderate loss of force at 24 and $72 \mathrm{~h}$ observed in the current study confirms that the prescribed lower-body resistance exercise caused EIMD. Although not indicative of myofibrillar disruption $[7,8]$, the small to very large increases in muscle soreness and CK activity indicate that tissue damage occurred after squatting exercise. The losses in MVC support previous observations of isometric strength loss after lower-body eccentric exercise in younger resistance trained males [21]. The reductions in MVC at $24 \mathrm{~h}$ possibly owe to both peripheral and central impairments, given the contemporaneous decrements in resting doublet and VA. However, that resting doublet scores were recovered by $72 \mathrm{~h}$, but VA remained suppressed, suggests that the reductions in MVC at the later time point were caused by central alterations. Potential central mechanisms include a reduction in drive to the muscle caused by neural impairments and reduction in excitability to the alpha motor-neuron [28,33]. 


\subsection{Changes in Indirect Markers of EIMD in Trained Young and Middle-Aged Males}

That differences between trained groups on plasma CK activity after resistance exercise were unclear reaffirms the findings of previous studies $[15,18,34]$, suggesting that membrane permeability is similar between trained young and middle age groups. Likewise, the comparable changes in muscle soreness observed in the two resistance trained groups is consistent with the work of Buford et al. [18], albeit in a non-resistance trained sample, in the plantar flexors, though contradictory to reports of greater soreness experienced by younger males after muscle-damaging elbow flexor exercise $[14,19]$. Increases in muscle soreness might reflect damage to connective tissue and decreases in range of motion, rather than damage to the contractile machinery per se [7,8]. Consequently, these data indicate that CK and muscle soreness responses to lower-limb muscle damaging exercise are similar in young and middle-aged resistance trained males.

\subsection{Changes in Muscle Function in Trained Young and Middle-Aged Males}

Reductions in MVC, VA, and resting doublet occurred in both resistance trained groups after EIMD. The finding that Pre VA values were not different between groups contrasts previous suggestions that older healthy adults are unable to activate the muscle to the same extent as their young counterparts [35], possibly owing to the trained nature of the MT group [36]. That the time course of VA recovery after high volume squatting exercise was not different between the MT and YG groups is also a novel finding. The moderately greater reductions in MVC in the MT group, compared to the YG group after EIMD, appear to be mediated by peripheral alterations (i.e., disruptions of sarcomeres and impaired excitation-contraction coupling), as reflected by the lower resting doublet values in the older trained participants. Given that differences in VA were unclear between the resistance trained groups after EIMD suggests that central alterations are not responsible for the greater reductions in MVC in the MT group.

The lower Pre peak power values at 20\% and $80 \% 1 \mathrm{RM}$ in the MT group, compared to the YG group, are similar to those previously reported in resistance trained middle-aged males [5]. For the first time, this study has highlighted that the decrements in peak power after EIMD are of a greater magnitude in middle-aged males, compared to young resistance trained males. Work in young athletes indicates that lower-body power output has strong relationships with a variety of sporting tasks [37,38]. Thus, it is plausible that the impaired power output due to EIMD may inhibit these movements in trained young and middle-aged males. Applied practitioners should therefore be cognisant of this and consider adopting different recovery practices for young and middle-aged male athletes after muscle-damaging lower-limb exercise.

\subsection{Differences in Recovery Between Trained and Untrained Middle-Aged Males}

The two middle-aged groups produced similar peak power during the muscle-damaging protocol, which was followed by similar changes in MVC, VA, resting doublet, and CK. The repeated bout effect (RBE) $[7,10]$ suggests that resistance trained males should experience less muscle damage after eccentric exercise compared to untrained males. However, the attenuated protection offered to the muscle with ageing $[12,13]$ might explain the similar recovery profiles in these age groups. Moreover, the similar sporting characteristics of the two middle-aged groups might also explain why both demonstrated a comparable recovery profile. That is, the training experienced by both groups during their sports participation might have provided a similar protection to the muscle-damaging squatting exercise. A further explanation might be owed to the similar peak power produced during the muscle-damaging protocol. It has been noted previously that the magnitude of EIMD and recovery were positively related to the workload during the muscle damaging protocol in young and older adults [39]. Given that both middle-aged groups produced a similar peak power during the exercise protocol, it is perhaps not unexpected that the recovery profile was similar. After high volume squatting, differences between middle-aged groups in perceived muscle soreness and peak power were moderate to large. After 
muscle damaging exercise, the MU group demonstrated greater losses in peak power compared to the MT group. It is plausible that the resistance training experience of the MT group served to preserve or enhance the type 2 fiber cross-sectional area [40], thus accounting for their smaller losses in peak power. Consequently, resistance training in middle-aged males might help to maintain lower-body peak power after muscle-damaging exercise, but does not appear to alter other indirect markers of EIMD.

\subsection{Limitations}

Readers should be aware of the cross-sectional nature of this study. That is, cause and effect cannot directly be established, but rather, only associations between age groups and different training status. However, given the large differences between age groups ( $>18$ years), designing a study that spanned over $\sim 18$ years would be unfeasible. Whilst the high variability in plasma CK in our sample is concerning, it should be noted that CK alterations show a poor temporal pattern with muscle function [41]. As such, the CK alterations should be used to confirm tissue damage, rather than indicate the magnitude of muscle damage.

\section{Conclusions}

This study reports that the magnitude of EIMD, as indicated by a reduction in muscle function, and time-course of recovery after high volume resistance exercise is greater in trained middle-aged males compared to their young counterparts. Practically, trained middle-aged males should be cognisant of requiring greater recovery time and adopt appropriate strategies. Moreover, resistance training in middle-aged males could attenuate the losses in peak power after high volume squatting exercise, but does not alter the recovery profile of other indirect markers of muscle damage. Applied practitioners should be mindful of these alterations in trained and untrained middle-aged males and should programme training accordingly.

Author Contributions: Conceptualization, J.F.T.F., K.L.L. and C.T.; Methodology, J.F.T.F., K.L.L. and C.T.; Formal Analysis, J.F.T.F.; Investigation, J.F.T.F.; Resources, J.F.T.F.; Data Curation, J.F.T.F.; Writing-Original Draft Preparation, J.F.T.F.; Writing—Review \& Editing, J.F.T.F., K.L.L. and C.T.; Supervision, K.L.L. and C.T.

Funding: This research received no external funding.

Conflicts of Interest: There are no conflict of interest.

\section{References}

1. Office for National Statistics. National Population Projections: 2014-Based Statistical Bulletin. Available online: https://www.ons.gov.uk/peoplepopulationandcommunity/populationandmigration/ populationprojections/bulletins/nationalpopulationprojections/2015-10-29 (accessed on 28 May 2019).

2. Pantoja, P.D.; Saez De Villarreal, E.; Brisswalter, J.; Peyré-Tartaruga, L.A.; Morin, J.B. Sprint acceleration mechanics in masters athletes. Med. Sci. Sports Exerc. 2016, 48, 2469-2474. [CrossRef] [PubMed]

3. Frontera, W.R.; Suh, D.; Krivickas, L.S.; Hughes, V.A.; Goldstein, R.; Roubenoff, R. Skeletal muscle fiber quality in older men and women. Am. J. Physiol. Cell Physiol. 2000, 279, C611-C618. [CrossRef] [PubMed]

4. Candow, D.G.; Chilibeck, P.D. Differences in size, strength, and power of upper and lower body muscle groups in young and older men. J. Gerontol. Biol. Sci. 2005, 60, 148-156. [CrossRef]

5. Fernandes, J.F.T.; Lamb, K.L.; Twist, C. A comparison of load-velocity and load-power relationships between well-trained young and middle-aged males during three popular resistance exercises. J. Strength Cond. Res. 2018, 32, 1440-1447. [CrossRef]

6. Roth, S.M.; Martel, G.F.; Ivey, F.M.; Lemmer, J.T.; Tracy, B.L.; Hurlbut, D.E.; Metter, E.J.; Hurley, B.F.; Rogers, M.A. Ultrastructural muscle damage in young vs. older men after high-volume, heavy-resistance strength training. J. Appl. Physiol. 1999, 86, 1833-1840. [CrossRef]

7. Hyldahl, R.D.; Hubal, M.J. Lengthening our perspective: Morphological, cellular, and molecular responses to eccentric exercise. Muscle Nerve 2014, 49, 155-170. [CrossRef]

8. Damas, F.; Nosaka, K.; Libardi, C.A.; Chen, T.C.; Ugrinowitsch, C. Susceptibility to exercise-induced muscle damage: A cluster analysis with a large sample. Int. J. Sports Med. 2016, 37, 633-640. [CrossRef] [PubMed] 
9. Machado, M.; Willardson, J.M. Short recovery augments magnitude of muscle damage in high responders. Med. Sci. Sports Exerc. 2010, 42, 1370-1374. [CrossRef]

10. Hyldahl, R.D.; Chen, T.C.; Nosaka, K. Mechanisms and mediators of the skeletal muscle repeated bout effect. Exerc. Sport Sci. Rev. 2017, 45, 24-33. [CrossRef]

11. Nosaka, K.; Sakamoto, K.E.I.; Newton, M.; Sacco, P. How long does the protective effect on eccentric exercise-induced muscle damage last? Med. Sci. Sports Exerc. 2001, 33, 1490-1495. [CrossRef] [PubMed]

12. Lavender, A.P.; Nosaka, K. Responses of old men to repeated bouts of eccentric exercise of the elbow flexors in comparison with young men. Eur. J. Appl. Physiol. 2006, 97, 619-626. [CrossRef]

13. Gorianovas, G.; Skurvydas, A.; Streckis, V.; Brazaitis, M.; Kamandulis, S.; McHugh, M.P. Repeated bout effect was more expressed in young adult males than in elderly males and boys. Biomed. Res. Int. 2013, 2013. [CrossRef] [PubMed]

14. Lavender, A.P.; Nosaka, K. Comparison between old and young men for changes in makers of muscle damage following voluntary eccentric exercise of the elbow flexors. Appl. Physiol. Nutr. Metab. 2006, 31, 218-225. [CrossRef]

15. Lavender, A.P.; Nosaka, K. Fluctuations of isometric force after eccentric exercise of the elbow flexors of young, middle-aged, and old men. Eur. J. Appl. Physiol. 2007, 100, 161-167. [CrossRef] [PubMed]

16. Gordon, J., III; Hoffman, J.R.; Arroyo, E.; Varanoske, A.; Coker, N.; Gepner, Y.; Wells, A.; Stout, J.; Fukuda, D. Comparisons in the recovery response from resistance exercise between young and middle-aged men. J. Strength Cond. Res. 2017, 31, 3454-3462. [CrossRef] [PubMed]

17. Lavender, A.P.; Nosaka, K. Changes in markers of muscle damage of middle-aged and young men following eccentric exercise of the elbow flexors. J. Sci. Med. Sport 2008, 11, 124-131. [CrossRef]

18. Buford, T.W.; MacNeil, R.G.; Clough, L.G.; Dirain, M.; Sandesara, B.; Pahor, M.; Manini, T.M.; Leeuwenburgh, $\mathrm{C}$. Active muscle regeneration following eccentric contraction-induced injury is similar between healthy young and older adults. J. Appl. Physiol. 2014, 116, 1481-1490. [CrossRef] [PubMed]

19. Chapman, D.W.; Newton, M.; McGuigan, M.R.; Nosaka, K. Comparison between old and young men for responses to fast velocity maximal lengthening contractions of the elbow flexors. Eur. J. Appl. Physiol. 2008, 104, 531-539. [CrossRef]

20. Batterham, A.; George, K. Reliability in evidence-based clinical practice: A primer for allied health professionals. Phys. Sport 2003, 4, 122-128. [CrossRef]

21. Macdonald, G.Z.; Button, D.C.; Drinkwater, E.J.; Behm, D.G. Foam rolling as a recovery tool after an intense bout of physical activity. Med. Sci. Sports Exerc. 2014, 46, 131-142. [CrossRef]

22. Batterham, A.M.; Atkinson, G. How big does my sample need to be? A primer on the murky world of sample size estimation. Phys. Sport 2005, 6, 153-163. [CrossRef]

23. Fernandes, J.F.T.; Lamb, K.L.; Twist, C. The intra- and inter-day reproducibility of the FitroDyne as a measure of multi-jointed muscle function. Isokinet. Exerc. Sci. 2016, 24, 39-49. [CrossRef]

24. Jackson, A.S.; Pollock, M.L. Generalized equations for predicting body density of men. Br. J. Nutr. 1978, 40, 497-504. [CrossRef] [PubMed]

25. Heyward, V.H.; Wagner, D.R. Applied Body Composition Assessment; Human Kinetics: Champaign, IL, USA, 2004.

26. Wathen, D. Load Assingment. In Essenetials of Strength and Conditioning; Human Kinetics: Champaign, IL, USA, 1994; pp. 435-446.

27. LeSuer, D.; McCormick, J.; Mayhew, J.; Wasserstein, R.; Arnold, M. The accuracy of prediction equations for estimating 1-RM performance in the bench press squat and deadlift. J. Strength Cond. Res. 1997, 11, 211-213.

28. Morton, J.P.; Atkinson, G.; MacLaren, D.P.M.; Cable, N.T.; Gilbert, G.; Broome, C.; McArdle, A.; Drust, B. Reliability of maximal muscle force and voluntary activation as markers of exercise-induced muscle damage. Eur. J. Appl. Physiol. 2005, 94, 541-548. [CrossRef] [PubMed]

29. Burt, D.G.; Lamb, K.; Nicholas, C.; Twist, C. Effects of exercise-induced muscle damage on resting metabolic rate, sub-maximal running and post-exercise oxygen consumption. Eur. J. Sport Sci. 2014, 14, 337-344. [CrossRef] [PubMed]

30. Hopkins, W.G.; Marshall, S.W.; Batterham, A.M.; Hanin, J. Progressive statistics for studies in sports medicine and exercise science. Med. Sci. Sports Exerc. 2009, 41, 3-12. [CrossRef]

31. Batterham, A.M.; Hopkins, W.G. Making meaningful inferences about magnitudes. Int. J. Sports Physiol. Perform. 2006, 1, 50-57. [CrossRef] 
32. Cohen, J. Statistical Power Analysis for the Behavioral Science; Lawrence Earlbaum Associates: Hilsdale, NJ, USA, 1988.

33. Avela, J.; Kyröläinen, H.; Komi, P.V.; Rama, D. Reduced reflex sensitivity persists several days after long-lasting stretch-shortening cycle exercise. J. Appl. Physiol. 1999, 86, 1292-1300. [CrossRef]

34. Manfredi, T.G.; Fielding, R.A.; O'Reilly, K.; Meredith, C.N.; Lee, Y.; Evans, W.J. Plasma creatine kinase actiivty and eimd in older men. Med. Sci. Sports Exerc. 1991, 23, 1028-1034. [CrossRef]

35. Klass, M.; Baudry, S.; Duchateau, J. Voluntary activation during maximal contraction with advancing age: A brief review. Eur. J. Appl. Physiol. 2007, 100, 543-551. [CrossRef]

36. Knight, C.A.; Kamen, G. Adaptations in muscular activation of the knee extensor muscles with strength training in young and older adults. J. Electromyogr. Kinesiol. 2001, 11, 405-412. [CrossRef]

37. Cronin, J.B.; Hansen, K.T. Strength and power predictors of sports speed. J. Strength Cond. Res. 2005, 19, 349-357.

38. Delaney, J.A.; Scott, T.J.; Ballard, D.A.; Duthie, G.M.; Hickmans, J.A.; Lockie, R.G.; Dascombe, B.J. Contributing factors to change-of-direction ability in professional rugby league players. J. Strength Cond. Res. 2015, 29, 2688-2696. [CrossRef] [PubMed]

39. Toft, A.D.; Jensen, L.B.; Bruunsgaard, H.; Ibfelt, T.; Halkjaer-Kristensen, J.; Febbraio, M.; Pedersen, B.K. Cytokine response to eccentric exercise in young and elderly humans. Am. J. Physiol. Cell Physiol. 2002, 283, C289-C295. [CrossRef] [PubMed]

40. Verdijk, L.B.; Gleeson, B.G.; Jonkers, R.A.M.; Meijer, K.; Savelberg, H.H.C.M.; Dendale, P.; Van Loon, L.J.C. Skeletal muscle hypertrophy following resistance training is accompanied by a fiber type-specific increase in satellite cell content in elderly men. J. Gerontol. Ser. A Biol. Sci. Med. Sci. 2009, 64, 332-339. [CrossRef] [PubMed]

41. Friden, J.; Lieber, R.L. Eccentric exercise-induced injuries to contractile and cytoskeletal muscle fibre components. Acta Physiol. Scand. 2001, 171, 321-326. [CrossRef]

(C) 2019 by the authors. Licensee MDPI, Basel, Switzerland. This article is an open access article distributed under the terms and conditions of the Creative Commons Attribution (CC BY) license (http://creativecommons.org/licenses/by/4.0/). 